\title{
Influence of altitude on seed and seedling characteristics in Quercus leucotrichophora A. Camus. ex. Bahadur
}

\author{
By K. P. Saklani ${ }^{1)}$, Bhupendra Singh ${ }^{1)}$ and B. P. BhatT ${ }^{2)}$ \\ ICAR Research Complex for Eastern Region, ICAR Parisar, P.O.-B.V. College, \\ Patna-800 014, Bihar w.e.f. 01.01.2011
}

(Received 22 ${ }^{\text {th }}$ June 2011)

\begin{abstract}
The present investigation was conducted to record variation of seed and seedling morphological characteristics and germination among eighteen provenances of Quercus leucotrichophora. These traits are important for improving the planting stock material in hand. Significant variations $(\mathrm{p}<0.05)$ were recorded for different morphological parameters of seed and seedling among the different provenances. Seed weight was the most variable character among morphological characteristics. Shoot/root ratio and root growth were more variable characters among the seedling traits. Altitude had significant positive relationship with seed weight, seed length and germination per cent. Soil $\mathrm{pH}$ was positively correlated with seed weight and germination. These parameters could be used as important criteria for seed source selection. Broad-sense heritability $\left(\mathrm{h}^{2}\right)$ calculated for different seed and seedling traits showed a considerable portion of genetic variation suggesting the possibility of rapid genetic improvement of these traits. Furthermore, this variability appeared to be geographically structured and would be mainly genetically controlled. Thus the results of present study should be helpful for growing planting material of this potential tree species in afforestation of subtropical to temperate region of Garhwal Himalaya, India.
\end{abstract}

Key words: Seed origin, seed dimensions, genetic variability, heritability, Quercus leucotrichophora seedling, growth characteristics.

\section{Introduction}

Quercus leucotrichophora A. Camus. ex. Bahadur is a moderate-sized to large evergreen tree attaining a height of $12-18 \mathrm{~m}$, belonging to family Fagaceae (GAUR, 1999). $Q$. leucotrichophora is the commonest oak of the western Himalaya, extending eastward to Nepal, within altitude range from 1000 to $2400 \mathrm{~m}$ asl (above sea level) but occasionally descending lower in moist situations (NEGI and NAITHANI, 1995). In Garhwal Himalaya, there are a number of indigenous tree species used as fuelwood, fodder and small timber by local people. However, with substantial increase in human and bovine population coupled with decreasing forests, people of the region are facing acute scarcity of fuel, fodder and timber (SACHAN, 2007). Q. leucotrichophora is the commonest

\footnotetext{
1) Department of Forestry, Post Box-59, H.N.B. Garhwal University, Srinagar Garhwal, Uttarakhand, India.

2) Author for correspondence: B. Р. Внатт. Phone: $+0612-2223962$ Cell: 094731-99366; Fax: +91-612-2223956.

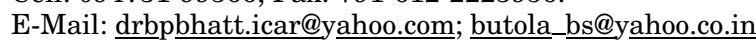

oak of Central Himalaya, India, facing the anthropogenic pressure. Its regeneration is very poor partly due to over exploitation and partly due to damage of seed populations by insect (Calandra sculpturata) and Himalayan langoor (Presbytis entellus). Mast seed year in $Q$. leucotrichophora takes place in interval of every 2-3 years, which may be another reason for its poor regeneration. Establishment of plantations of this native multipurpose tree species is recommended as suitable conservation strategy. In this Himalayan region, lack of quality planting material could be improved by selecting more suitable provenance.

Despite its great importance, the efforts for the genetic improvement of $Q$. leucotrichophora are scanty. Populations of a species from different geographical locations (provenances) frequently show differential response due to genotype effect (MWASE et al., 2006; ANDERSON et al., 2008; SAIKIA et al., 2009; SingH et al., 2010). Seed size and weight of several species varied among provenances (Dhanai et al. 2003; Singh et al., 2006; ZHEng et al., 2009; GHILDIYAL et al., 2009), as well as germination (GINWAL et al., 1996; CHAUHAN et al., 1996; UNIYAL et al., 2002; SinGH et al., 2004) and growth and biomass performances (IsIK, 1986; Holm, 1994; CHAUHAN et al., 1996; SingH et al., 2006). Variation in seed and seedling growth between provenances have been observed in different tree species, but such information is lacking for $Q$. leucotrichophora. To mitigate the growing demand of this species, there is a need of selection of best provenance of $Q$. leucotrichophora so as to improve its productivity in agroforestry systems as well as in various afforestation programmes in Central Himalaya, India.

\section{Material and Methods}

Mature seeds of $Q$. leucotrichophora were collected from a wide altitudinal range within their natural distribution. The word seed/seeds refers to the true seed with the pericarp (true seed + pericarp $=$ an acorn). For each site, seeds were collected from 10 randomly selected healthy and mature standing trees in the month of December. Each tree was $100 \mathrm{~m}$ apart from each other to avoid narrowing down the variation sampled due to relatedness (ScHMIDT, 2000). Seeds were collected from eighteen provenances of Garhwal hills and Himanchal Pradesh (H.P.) comprising the central parts of Himalaya, India. Geographical location of seed source ranged from $29^{\circ} 19^{\prime} \mathrm{N}$ to $30^{\circ} 57^{\prime} \mathrm{N}$ latitude and $78^{\circ} 3^{\prime} \mathrm{E}$ to $79^{\circ} 55^{\prime} 55^{\prime} \mathrm{E}$ longitude. The altitude of the entire range varied from 1015 to $2400 \mathrm{~m}$ asl. (Table 1, Figure 1). Soil 
Table 1. - Geographical origin of Q. leucotrichophora seed sampling locations and soil $\mathrm{pH}$ value.

\begin{tabular}{|c|c|c|c|c|c|c|}
\hline Provenances & Districts & $\begin{array}{l}\text { Altitude } \\
\text { (m asl) }\end{array}$ & Latitude (N) & Longitude (E) & $\begin{array}{c}\text { Rainfall } \\
\text { (mmi) }\end{array}$ & Soil pll \\
\hline Dovsrikot & Pauri & 1015 & $30^{\prime \prime} 10^{\prime}$ & $78^{\circ} 47^{\prime} 8^{\prime \prime}$ & 965.0 & 5.84 \\
\hline Bankura & Pauri & 1100 & $29^{11} 55^{\prime}$ & $79^{11} 2^{\prime} 10^{\prime \prime}$ & 996.0 & 5.86 \\
\hline Baijron & Pauri & 1350 & $29^{\mathrm{d}} 55^{\prime} 20^{\circ}$ & $79^{01} 2^{\prime} 50^{\prime \prime}$ & 1065.9 & 5.82 \\
\hline Rampunga & Chamoli & 1400 & $30^{\circ} 35^{\circ}$ & $79^{\prime \prime} 2^{\prime} 55^{\prime \prime}$ & 1620.3 & 5.80 \\
\hline Budashu & Pauri & 1450 & $30^{\circ} 11^{\prime} 30^{\circ}$ & $78^{0} 53^{\prime}$ & 989.0 & 5.82 \\
\hline Mandal & Chamoli & 1600 & $30^{0} 26^{\prime}$ & $79^{0} 16^{\prime}$ & 1800.0 & 6.18 \\
\hline Solan & Solan & 1650 & $30^{10} 57^{1}$ & $79^{11} 5^{\prime}$ & 1095.0 & 5.97 \\
\hline Bersuri & Pauri & 1650 & $29^{\circ} 55^{\prime} 40^{\circ}$ & $79^{0} 5^{\prime} 55^{11}$ & 1268.5 & 5.89 \\
\hline Jamu & Rudraprayag & 1700 & $30^{\circ} 34^{\prime} 35^{\circ}$ & $79^{11} 2 \cdot 45^{11}$ & 1750.97 & 6.07 \\
\hline Kandoliya & Pauri & 1700 & $30^{\circ} 8^{\prime}$ & $78^{\prime \prime} 46^{\prime} 30^{\prime \prime}$ & 1066.0 & 5.92 \\
\hline Khirshu & Pauri & 1800 & $30^{01} 11^{1}$ & $78^{0} 52^{\prime}$ & 1099.6 & 5.99 \\
\hline Randhar & Tehri & 1830 & $30^{6} 22^{\prime} 45^{\prime \prime}$ & $78^{0} 55^{\prime}$ & 1497.23 & 6.08 \\
\hline Voranibeta & Chamoli & 2000 & $30^{6} 34^{\prime} 10^{\circ}$ & $79^{1 \mathrm{II}} 2^{\prime} 20^{\prime \prime}$ & 1536.0 & 5.62 \\
\hline Damta & Uttarkashi & 2020 & $30^{\circ} 38^{\prime}$ & $78^{11} 3^{\prime}$ & 1387.0 & 6.15 \\
\hline Gumkhal & Pauri & 2025 & $29^{0} 55^{1}$ & $78^{0} 38^{\prime}$ & 1065.0 & 6.30 \\
\hline Kasturgamirgbihar & Chamoli & 2150 & $30^{11} 28^{\prime}$ & $79^{11} 55^{\prime} 55^{\prime \prime}$ & 1800.0 & 6.24 \\
\hline Bunda & Tehri & 2300 & $30^{\circ} 30^{\prime} 15^{\prime \prime}$ & $78^{0} 55^{\prime} 55^{\prime \prime}$ & 1790.0 & 6.32 \\
\hline Chaurangikhal & Utlarkashi & 2400 & $29^{\circ 1} 19^{\prime}$ & $79^{\circ} 38^{\prime}$ & 1769.0 & 6.19 \\
\hline
\end{tabular}

$\mathrm{pH}$ was measured by Kelway Soil Tester directly in the field (Table 1).

From each provenance bulk seeds sample was collected and brought to the laboratory and allowed to dry in shade for $24 \mathrm{~h}$. The sample collected from each provenance was then placed in a cotton bag, allotted an accession number (name of place), and stored in a cooling incubator for further experiments.

\section{Seed Morphology}

The moisture percentage of each seed sample was determined on a fresh weight basis by drying the seeds at $103 \pm 3^{\circ} \mathrm{C}$ as per ISTA (1999) rules. Seed length and breadth (in the middle portion of the seed) were measured on five replicate samples, each consisting of 100 seeds randomly drawn from each seed lot using micrometer (Besto make). Seed weight (g/100 seed) was recorded by choosing eight random samples of each population, i.e., 100 seeds per seed lot (ISTA, 1999). Five samples (each of 100 seeds) were drawn from seed lot of each provenance and numbers of seeds damaged due to insect attack and healthy seeds were determined.

\section{Seed germination and seedling growth traits}

Eight replicates (of 100 seeds each) were used for germination. Petri dishes (12 cm diameter) were placed in the germinators pre fixed at $25^{\circ} \mathrm{C}$ constant temperature regimes. Distilled water $(1 \mathrm{ml})$ was used daily to moisten Whatman filter paper in germinator. Radicle protrusion was used as the indicator of the germination. Germination per cent was the percent of the sown seeds germinated at the completion of test period, i.e., 28 days after sowing (ISTA, 1999). Germination value was also calculated according to CzABATOR (1962) as $\mathrm{GV}=\mathrm{PV} \times \mathrm{MDG}$, where, $\mathrm{GV}=$ germination value; $\mathrm{PV}=$ the peak value of germination, and $\mathrm{MDG}=$ mean daily germination.

Germinating seeds of each population were transferred into polythene bags (1 $\mathrm{kg}$ volume) containing sand, soil and farmyard manure in 1:2:1 ratio and placed in the experimental garden of Forestry Department, Srinagar, Uttarakhand, India (situated between $30^{\circ} 13^{\prime} \mathrm{N}$ latitude and $78^{\circ} 48^{\prime} \mathrm{E}$ longitude at an elevation of $530 \mathrm{~m}$ asl). The seedlings of each population (four replicates, 25 each) were randomly tagged and maintained in nursery for one year. At the end of one year's growth, a tagged seedlings were uprooted and assessed for their shoot length, root length, collar diameter and number of leaves/plant was counted. Root length was divided by the shoot length of the same seedling to

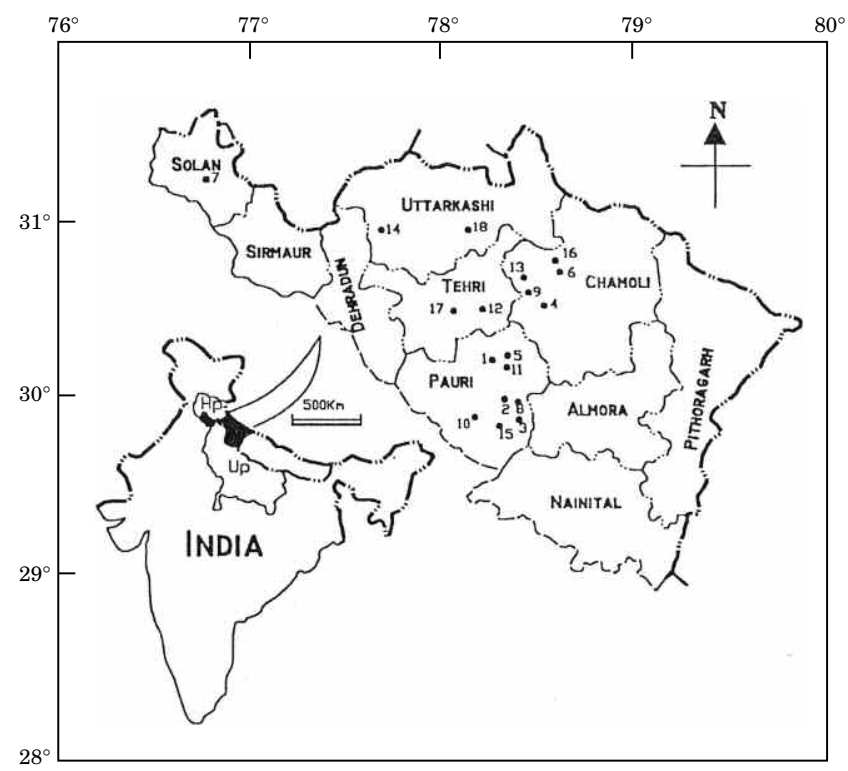

Figure 1. - Seed collection sites (Provenances) of Quercus leucotrichophora.

1. Dov Srikot; 2. Bankura; 3. Baijron; 4. Ranpunga; 5. Budashu; 6. Mandal; 7. Solan; 8. Bersuni; 9. Jamu; 10. Kandoliya; 11. Khirshu; 12. Randhar; 13. Voranibeta; 14. Damta; 15. Gumkhal; 16. Kasturgamirgbihar; 17. Budna; 18. Chaurangikhal. 
determine the root/shoot length ratio. These 100 seedlings of each provenance were divided into root, shoot and leaves, and oven dried for a period of $48 \mathrm{~h}$ at $98 \pm 2{ }^{\circ} \mathrm{C}$ before determining their dry weight.

\section{Statistical Analysis}

The seed and seedling attributes were statistically analyzed by SPSS software package, version 10.0.1 (SPSS Inc., Chicago, USA) for analysis of variance, and correlation coefficients to record the effect of altitude on germination and growth performance of seedlings. Tukey-test (BARTZ, 1988) was used to estimate the variation of seed, seedling growth and germination parameters with provenance. Broad sense heritability and genetic gain were determined as suggested by JOHNSON et al. (1955).

\section{Results}

\section{Morphological studies}

Among provenances, seed length ranged from 1.98 (Baijron) to $2.65 \mathrm{~cm}$ (Kasturgamrigbihar) and breadth extended from 12.28 (Solan) to $15.53 \mathrm{~mm}$ (Kandoliya). Similarly, seed weight ranged from 107.0 to $258.33 \mathrm{~g} / 100$ seed. On average, there was $1.33,1.26$ and 2.41-fold variation between highest and lowest values of seed length, breadth and weight, respectively, irrespective of provenances. Among various morphological characters, maximum variations were recorded in seed weight and minimum in seed breadth. The maximum (98.0\%) healthy seed was affirmed in Bersuri and lowest (62.0\%) in Budashu provenance. Similarly, maximum (38.0\%) damaged seed was recorded in Budashu and minimum $(2.0 \%)$ in Barsuri provenance.

Coefficient of variation (CV) showed that all the characters were not equally variable and it was highest
$(63.48 \%)$ to damaged seeds and lowest $(5.85 \%)$ variation was recorded in seed breadth (Table 2 ).

\section{Germination}

Used temperature accelerated optimal germination in almost all the populations, however, the germination per cent ranged from 77.0 (Ranpunga) to $100.0 \%$ (Mandal) among provenances. The germination value ranged from 0.77 to 1.83 among the provenances. Coefficient of variation for germination value and germination per cent was 28.40 and 6.28, respectively (Table 3 ).

\section{Growth studies}

Survival per cent of seedlings in nursery ranged from 78.5 (Dovsrikot) to 95.5 (Solan) in different provenances. Maximum (95.08\%) plantable seedling was recorded in Budashu and minimum $(62.15 \%)$ in Randhar provenance (Table 3).

Shoot length was the highest in Budashu $(39.55 \mathrm{~cm})$ and the lowest $(31.81 \mathrm{~cm})$ in Dovsrikot provenance. Likewise, root length was maximum $(41.86 \mathrm{~cm})$ in Bankura and minimum $(15.43 \mathrm{~cm})$ in Bersuri among various provenances. Root/shoot ratio was recorded maximum (1.14) in Bankura and minimum (0.47) in Solan provenance. Collar diameter of seedlings was recorded maximum $(3.80 \mathrm{~mm})$ in Bersuri provenance and minimum $(2.76 \mathrm{~mm})$ in Solan provenance. Maximum (12.44) number of leaves per plant was recorded in Kandoliya provenance. Among various components of seedlings, roots contributed the highest biomass followed by leaves and shoot, irrespective of provenances. On average, there were $4.62,1.97$ and 2.86 -fold variations between minimum and maximum values of biomass partitioned by roots, shoot and leaves, respectively, irrespective of provenances. However, among various provenances, seedlings of Kandoliya exhibited the high-

Table 2. - Provenance variation in different seed characteristics in $Q$. leucotrichophora. Values in the same column with the same letter do not differ significant $(\mathrm{p}<0.05)$.

\begin{tabular}{|c|c|c|c|c|c|c|}
\hline Provenances & $\begin{array}{c}\text { Altitude } \\
\text { (m as } 1)\end{array}$ & $\begin{array}{l}\text { Length } \\
\text { (cm) }\end{array}$ & $\begin{array}{c}\text { Breadth } \\
(\mathrm{mm})\end{array}$ & $\begin{array}{c}\text { Weight } \\
\text { (g/100acorn) }\end{array}$ & lealthy seeds \% & $\begin{array}{c}\text { Daniaged } \\
\text { seeds } \%\end{array}$ \\
\hline Dovsrikot & 1015 & $2.01^{\mathrm{ii}}$ & $13.46^{\mathrm{wex}}$ & $107.00^{\mathrm{i}}$ & $73.0^{m}$ & $27.0^{\mathrm{hc}}$ \\
\hline Bankura & 1100 & $2.06^{\mathrm{hi}}$ & $13.03^{\mathrm{hi}}$ & $125.00^{\mathrm{rgh}}$ & $80.0^{\mathrm{cec}}$ & $20.0^{i c \Gamma^{\circ}}$ \\
\hline Baijron & 1350 & $1.98^{i}$ & $12.37^{\mathrm{H}}$ & $122.66^{y_{1}}$ & $84.0^{\text {cdt }}$ & $16.0^{0.5 !}$ \\
\hline Ranpunga & 1400 & $2.46^{\mathrm{c}}$ & $13.74^{\text {cocey }}$ & $159.33^{\mathrm{k} k}$ & $85.0^{\text {ct }}$ & $15.0^{\mathrm{fg}}$ \\
\hline Budashu & 1450 & $2.00^{\mathrm{ij}}$ & $13.70^{\mathrm{de} f y}$ & $131.33^{\text {rgh }}$ & $62.0^{\mathrm{i}}$ & $38.0^{\mathrm{a}}$ \\
\hline Mandal & 1600 & $2.09^{\mathrm{Ehi}}$ & $14.12^{\mathrm{bc}}$ & $186.66^{\circ}$ & $97.0^{13}$ & $3.00^{i}$ \\
\hline Solan & 1650 & $2.17^{\mathrm{efi} !}$ & $12.28^{1}$ & $132.33^{\text {ry }}$ & $78.0^{\mathrm{fg}}$ & $22.0^{\mathrm{cI}}$ \\
\hline Bersuri & 1650 & $2.14^{\text {git. }}$ & $13.39^{\text {tich }}$ & $146.00^{\mathrm{C}^{*}}$ & $98.0^{\mathrm{a}}$ & $2.0^{i}$ \\
\hline Јатาџ & 1700 & $2.56^{\mathrm{ab}}$ & $12,84^{\mathrm{ii}}$ & $133.33^{\text {lis }}$ & $68.0^{1}$ & $32.0^{\mathrm{b}}$ \\
\hline Kandoliya & 1700 & $2.24^{\text {des }}$ & $15.53^{\text {a }}$ & $196.66^{7}$ & $79.0^{5:}$ & $21.0^{6 x}$ \\
\hline Khirshu & 1800 & $2.25^{\mathrm{dc}}$ & $13.02^{\text {lii }}$ & $250.00^{\mathrm{a}}$ & $62.0^{\prime}$ & $38.0^{\text {a }}$ \\
\hline Randhar & 1830 & $2.44^{*}$ & $12.88^{i j}$ & $248.00^{a}$ & $79.0^{4: 2}$ & $21.0^{\mathrm{ket}}$ \\
\hline Voranibeta & 2000 & $2.48^{\mathrm{bc}}$ & $12.59^{\mathrm{k}}$ & $170.00^{\mathrm{cds}}$ & $84.0^{\text {ade }}$ & $16.0^{4 \sqrt{6}}$ \\
\hline Janta & 2020 & $2.32^{d}$ & $13.55^{\text {detg }}$ & $258.33^{\mathrm{a}}$ & $89.0^{h \mathrm{hc}}$ & $11.0^{\mathrm{g}-1}$ \\
\hline Gumkhal & 2025 & $2.04^{\mathrm{iI}}$ & $13.88^{\mathrm{LC}}$ & $205.00^{\mathrm{a}}$ & $79.0^{\mathrm{LE}}$ & $21.0^{\mathrm{LE}}$ \\
\hline Kasturgamirgbihar & 2150 & $2.65^{\mathrm{a}}$ & $14.39^{\mathrm{h}}$ & $253.33^{\mathrm{a}}$ & $94.0^{\text {al? }}$ & $6.0^{\mathrm{hi}}$ \\
\hline Bunda & 2300 & $2.19^{\mathrm{ef}}$ & $13.84^{\text {cete }}$ & $196.66^{7}$ & $95.0^{9}$ & $5.0^{1}$ \\
\hline Chaurangikhal & 2400 & $2.32^{d}$ & $13.34^{\text {gh }}$ & $185.00^{3 k^{2}}$ & $95.0^{a}$ & $5.0^{1}$ \\
\hline C.V. & & 9.17 & 5.85 & 28.02 & 13.67 & 63.48 \\
\hline
\end{tabular}

C.V. = Coefficient of variation. 
Table 3. - Variation in seed germination, germination value and survival among the provenances of $Q$. leucotrichophora.

\begin{tabular}{|c|c|c|c|c|}
\hline Proventunces & $\mathrm{G} \%$ & $\mathrm{GV}$ & Survival $\%$ & $\begin{array}{l}\text { Plantable } \\
\text { seedling (\%) }\end{array}$ \\
\hline Dovsrikot & $90.00^{\mathrm{cos}}$ & $0.83^{i}$ & $78.5^{i k}$ & $64.17^{\mathrm{i}}$ \\
\hline Bankura & $90.00^{\mathrm{cod}}$ & $1.14^{\text {te }}$ & $88.7^{\text {cdef }}$ & $80.67^{\text {A1 }}$ \\
\hline Baijron & $97.00^{n^{3}}$ & $1.10^{9}$ & $85.5^{\mathrm{etg}}$ & $85.58^{\text {cd }}$ \\
\hline Ranpunga & $77.00^{\circ}$ & $1.28^{\mathrm{D}}$ & $80.75^{\text {hiij }}$ & $90.83^{\mathrm{ah}}$ \\
\hline Budashu & $97.00^{23}$ & $0.86^{i}$ & $75.10^{k}$ & $95.08^{\mathrm{a}}$ \\
\hline Mandal & $100.00^{\mathrm{at}}$ & $0.94^{1}$ & $95.0^{\text {alb }}$ & $80.40^{\mathrm{I}}$ \\
\hline Solan & $93.00^{\mathrm{kc}}$ & $0.96^{\mathrm{Fi}}$ & $95.5^{\mathrm{a}}$ & $75.11^{\circ}$ \\
\hline Bersuri & $93.00^{\mathrm{ke}}$ & $0.91^{i !}$ & $90.8^{\mathrm{bcd}}$ & $85.21^{\text {cile }}$ \\
\hline Jamu & $87.00^{\mathrm{de}}$ & $1.51^{\circ}$ & $95.0_{\mathrm{ab}}$ & $70.17^{\mathrm{h}}$ \\
\hline Kandoliya & $87.00^{\mathrm{de}}$ & $1.20^{5^{5}}$ & $90.5^{\mathrm{cdd}}$ & $84.20^{\mathrm{def}}$ \\
\hline Khirshu & $93.00^{\mathrm{be}}$ & $0.95^{\mathrm{Fi}}$ & $85.0^{\mathrm{rgh}}$ & $85.73^{\mathrm{cd}}$ \\
\hline Randhar & $93.00^{1: 2}$ & $1.83^{4}$ & $92.0^{\text {ablec }}$ & $62.15^{i}$ \\
\hline Voranjbetal & $87.00^{\mathrm{de}}$ & $1.07^{\text {th }}$ & $92.0^{\mathrm{abc}}$ & $73.24^{t^{5.1}}$ \\
\hline 1)amtal & $97.00^{1+17}$ & $1.74^{\mathrm{b}}$ & $89.5^{\text {date }}$ & $69.43^{7}$ \\
\hline Gumkhal & $83.00^{\mathrm{e}}$ & $0.81^{5}$ & $84.0^{\lg ^{\mathrm{hi}} \mathrm{i}}$ & $89.56^{\mathrm{kc}}$ \\
\hline Kasturgatnirgbihar & $93.00^{\mathrm{iLC}}$ & $1.61^{\mathrm{hc}}$ & $80.0^{\mathrm{i}}$ & $84.68^{i k T}$ \\
\hline Bunda & $97.00^{\mathrm{a} 3}$ & $0.77^{k}$ & $90.0^{\mathrm{cd}}$ & $81.73^{\text {iet }}$ \\
\hline Chaurangikhal & $93.00^{\mathrm{b}=\mathrm{C}}$ & $1.34^{5}$ & $87.0^{\text {detes }}$ & $92.62^{\text {al }}$ \\
\hline C.V. & 6.28 & 28.02 & 6.80 & 11.90 \\
\hline
\end{tabular}

$\mathrm{G} \%=$ germination percent, $\mathrm{GV}=$ Germination value, Values in the same column with the same letter do not differ significant $(\mathrm{p}<0.05)$.

est total biomass (4.92 g/plant) and Gumkhal the lowest (2.20 g/plant) (Table 4).

For seedling growth traits, maximum coefficient of variation was recorded for root/shoot ratio (28.07), followed by root length $(26.67 \%)$. Number of leaves and collar diameter had the lowest coefficient of variation (Table 4).

\section{Correlation Coefficient}

Significant positive $(p<0.01)$ relationship was recorded for seed length-germination value, healthy seed- damage seed, seed weight-healthy seed, germination value-shoot dry weight, plantable seedling-shoot length, root length-root-shoot ratio, shoot dry weight-total dry weight, root dry weight-root/shoot dry weigh, root dry weight-total dry weight and leaves dry weight-total dry weight. Significant $(p<0.05)$ positive correlations were recorded between seed length-seed weight, seed lengthshoot dry weight, seed weight-germination, seed weightgermination value, germination value-total dry weight, survival-plantable seedlings, survival-shoot length, shoot length-root length and shoot dry weight-root-shoot dry weight. Significant $(\mathrm{p}<0.05)$ inverse correlation was

Table 4. - Seedlings growth of $Q$. leucotrichophora after one year at nursery stage.

\begin{tabular}{|c|c|c|c|c|c|c|c|c|c|c|}
\hline Provenance & $\begin{array}{l}\text { Shoot length } \\
\text { (cm) }\end{array}$ & $\begin{array}{l}\text { [2oot length] } \\
(\mathrm{cm})\end{array}$ & $\begin{array}{l}\text { Root Shoot } \\
\text { ratio }\end{array}$ & $\begin{array}{l}\text { Collar } \\
\text { diameter } \\
(\mathrm{mm})\end{array}$ & $\begin{array}{l}\text { Number of } \\
\text { l.caves }\end{array}$ & $\begin{array}{l}\text { Shoot dry } \\
\text { wright (gm) }\end{array}$ & $\begin{array}{l}\text { Root dry } \\
\text { neight } \\
\text { (gm) }\end{array}$ & $\begin{array}{l}\text { Root Shoot } \\
\text { dry weight } \\
\text { ratio }\end{array}$ & $\begin{array}{l}\text { I ceaves } \\
\text { dry } \\
\text { weight } \\
\text { ratio }\end{array}$ & $\begin{array}{l}\text { Tolal dry } \\
\text { ueight } \\
\text { (gm) }\end{array}$ \\
\hline Dowsrikot & $31.81^{i}$ & $26.30^{g h}$ & $0.83^{21}$ & $2.85^{\mathrm{il}}$ & $11.66^{b}$ & $0.51^{k}$ & $1.53^{\mathrm{wl}}$ & $3.00^{\mathrm{n}}$ & $0.89^{13}$ & $2.93^{\mathrm{j} \mathrm{k}}$ \\
\hline Bankura & $36.60^{\text {di: }}$ & $41.86^{! 1}$ & $1.14^{: 1}$ & $3.10^{p+1}$ & $10.66^{19}$ & $0.78^{1 / t^{*}}$ & $1,12^{\mathrm{gh}}$ & $1.44^{\mathrm{b}:}$ & $1,33^{15 x^{*}}$ & $3,23^{m !}$ \\
\hline Baijron & $38.15^{\ln 5}$ & 28.28 & $0.74^{-6}$ & $3.06^{*}$ & $10.00^{\prime \prime}$ & $0.83^{\mathrm{kid}}$ & $1.47^{\text {do }}$ & $1.77^{\sqrt[10]{\gamma}}$ & $1.24^{\mathrm{asc}}$ & $3.54^{\text {de }}$ \\
\hline Ranpunga & $36.56^{\mathrm{d} x}$ & $27.46^{\mathrm{I!nh}}$ & $0.75^{-5}$ & $2.89^{i}$ & $10.00^{!}$ & $0.86^{h o}$ & $1.68^{\prime \prime}$ & $1.95^{: 12}$ & $1.42^{h}$ & $3.96^{\circ}$ \\
\hline Budashu & $39.55^{2}$ & $23.76^{\mathrm{h}}$ & $0.600^{\mathrm{h}}$ & $3.19^{2:}$ & $1000^{\prime:}$ & $0.79^{\mathrm{dit}}$ & $1.27^{\mathrm{it}: !}$ & 1.61 & $1.44^{13}$ & $3.50^{\circ}$ \\
\hline Mandal & $34.32^{5}$ & $18.70^{\circ}$ & $0.54^{\mathrm{ti}}$ & $3.12^{\text {pgh }}$ & $11.11^{\mathrm{acL}}$ & $0.58^{\mathrm{i}}$ & $1.39^{\mathrm{dit} \mathrm{P}^{2}}$ & $2.40^{\mathrm{hec}}$ & $1.89^{\mathrm{7}}$ & $3.86^{\mathrm{co}}$ \\
\hline Solan & $33.50^{-15:}$ & $15.70^{\circ}$ & $0.47^{\mathrm{i}}$ & $2.76^{j}$ & $11.44^{6 x}$ & $0.75^{\mathrm{cI}}$ & $1.09^{9 \mathrm{~s}}$ & $1.45^{\mathrm{b}:}$ & $1.16^{\mathrm{cs}}$ & $3.00^{\text {phij }}$ \\
\hline Bersuri & $32.24^{11 i}$ & $15.43^{!}$ & $0.48^{i}$ & $3.80^{:}$ & $10.33^{5}$ & $0.67^{\text {whh }}$ & $1.41^{\mathrm{Sint}}$ & $2.10^{1 !}$ & $1.19^{4.655}$ & $3.27^{0 * ! !}$ \\
\hline Jamu & $31.95^{i}$ & $28.60^{\mathrm{r} !}$ & $0.90^{\text {dic }}$ & $3.22^{\mathrm{int}}$ & $11.11^{\text {nat }}$ & $0.95^{7}$ & $1.95^{h}$ & $2.05^{\circ}$ & $1.42^{h}$ & $4.32^{2}$ \\
\hline Kandoliya & 34.44 & $25.60^{2 h}$ & 0.74 & $3.12^{\mathrm{rath}}$ & $12.44^{i n}$ & $0.83^{\text {E.d }}$ & $2.35^{a}$ & $2.83^{\mathrm{a}}$ & $1.41^{h}$ & $4.92^{k}$ \\
\hline Khirshu & $36.38^{2}$ & $26.66^{\mathrm{rh}}$ & $0.73^{9}$ & $2.92^{i}$ & $10.33^{1 ! !}$ & $0.54^{\mathrm{ik}}$ & 1.26 & $2.33^{\text {lnd }}$ & $0.93^{h}$ & $2.73^{16}$ \\
\hline Ranathar & $32.00^{i}$ & $23.78^{h}$ & $0.74^{-6}$ & 3.17 & $10.00^{\prime}$ & $0.79^{\mathrm{dt}}$ & $1.92^{k}$ & $2.43^{\mathrm{b}}$ & $0.66^{i}$ & $3.37^{6 .}$ \\
\hline Voranibcta & $32.66^{11 i}$ & $32.44^{\mathrm{dc}}$ & $0.99^{\mathrm{ket}}$ & $3.24^{\mathrm{dc}}$ & $12.33^{i}$ & $0.71^{\Gamma \mathrm{g}}$ & $1.09^{\mathrm{gh}}$ & $1.54^{\underline{y}}$ & $1.08^{5}$ & $2.88^{\mathrm{ijk}}$ \\
\hline Damta & $33.00^{21: 1:}$ & $30.40^{2: "}$ & $0.92^{\text {eclk }}$ & $3.32^{\circ 6}$ & $10.00^{!}$ & $0.81^{\mathrm{ded}}$ & $1.23^{\mathrm{fs}}$ & $1.52^{\mathrm{y}}$ & $1.31^{\text {hel. }}$ & $3.35^{2:}$ \\
\hline Gumkhal & $32.55^{1 i i}$ & $27.80^{\mathrm{fr}}$ & $0.85^{\prime:}$ & $3.20^{\mathrm{c}}$ & $10.00^{\circ}$ & $0.48^{k}$ & $1.02^{h}$ & $2.13^{\mathrm{i}: \mathrm{t}^{2}}$ & $0.70^{i}$ & $2.20^{1}$ \\
\hline Kasturgamirgbihar & $39.00^{3 / 2}$ & $40.13^{\text {alt }}$ & $1.03^{\text {ith }}$ & $3.51^{3}$ & $11.11^{\text {trd }}$ & $0.84^{\operatorname{lic}}$ & $1.69^{\circ}$ & $2 .(k)^{4}$ & $1.39^{h}$ & $3.92^{\circ}$ \\
\hline Budna & $37.78^{\text {lidd }}$ & $36.00^{\circ}$ & $0.95^{\text {le:iti }}$ & $3.177^{\text {ven: }}$ & $11.00^{\mathrm{d} x}$ & $0.63^{\mathrm{lij}}$ & $1.51^{\mathrm{dd}}$ & $2.40^{1 x:}$ & $1.32^{k}$ & $2.46^{\mathrm{N}}$ \\
\hline Chatrangikhal & $37.00^{\text {colke }}$ & $37.25^{\mathrm{bc}}$ & $\left.1.0\right|^{1: 1}$ & $3.25^{\text {de }}$ & $11.00^{\mathrm{de}}$ & $0.88^{b}$ & $1.07^{\mathrm{gh}}$ & $1.22^{i}$ & $1.17^{\mathrm{cet}}$ & $3.12^{\text {Ixphi }}$ \\
\hline C.V. & 7.59 & 26.67 & 28.07 & 7.60 & 7.39 & 18.65 & 24.98 & 24.74 & 24.13 & 19.76 \\
\hline
\end{tabular}

Values in the same column with the same letter do not differ significant $(\mathrm{p}<0.05)$. 
found between shoot length- root/shoot dry weights (Table 5a).

Altitude had significant positive relationship with seed weight $(\mathrm{p}<0.01)$, seed length, germination per cent and collar diameter of seedling $(\mathrm{p}<0.05)$. Latitude was significantly $(\mathrm{p}<0.05)$ negatively correlated with plantable seedling. Longitude had significant $(\mathrm{p}<0.05)$ positive correlation with shoot length. Rainfall was positively significantly $(\mathrm{p}<0.01)$ correlated with seed length and healthy seed. Damaged seed per cent was negatively correlated with rainfall. Rainfall was positively correlated with shoot dry weight and negatively correlated with root/shoot dry weight ratio. Soil $\mathrm{pH}$ of different provenance had significant $(\mathrm{p}<0.01)$ positive correlation with seed weight and germination (Table $5 b$ ).

\section{Heritability $\left(h^{2}\right)$ board sense:}

Heritability $\left(\mathrm{h}^{2}\right)$ broad sense was calculated the highest (92.26) for seed weight followed by germination value (91.67), and the lowest (10.00) for collar diameter. Seed length, seed breadth, seed germination, root length, root/shoot length ratio, number. of leaves/plant, shoot dry weight, root dry weight and leaves dry weight all showed moderately high heritability. Genetic gain

Table 5a. - Correlation coefficient between and among the various traits Q. leucotrichophora.

\begin{tabular}{|c|c|c|c|c|c|c|c|c|c|c|c|c|c|c|c|c|c|c|}
\hline Variatblat & 1 & 2 & 3 & 4 & 5 & 6 & 7 & 8 & 9 & 10 & $1]$ & 12 & 13 & 14 & 15 & 16 & 17 & 18 \\
\hline 2 & 0.04 & & & & & & & & & & & & & & & & & \\
\hline 3 & $0.47^{*}$ & 0.34 & & & & & & & & & & & & & & & & \\
\hline 4 & $\begin{array}{l}0.14 \\
-0.14\end{array}$ & $\begin{array}{r}0.22 \\
-0.22\end{array}$ & $\begin{array}{l}0.20 \\
-0.20\end{array}$ & $\stackrel{-}{1.00^{* * *}}$ & & & & & & & & & & & & & & \\
\hline is & -0.16 & 0.39 & $0.5]^{*}$ & 0.31 & -0.31 & & & & & & & & & & & & & \\
\hline 7 & $0.72 * *$ & -0.01 & $0.49 *$ & 0.12 & -0.12 & -0.06 & & & & & & & & & & & & \\
\hline 8 & 0.18 & -0.24 & 0.07 & 0.29 & -0.29 & 0.38 & 0.17 & & & & & & & & & & & \\
\hline 9 & -0.27 & 0.72 & $-(0.06$ & 0.10 & -0.10 & -0.15 & -0.38 & $0.4 .3^{*}$ & & & & & & & & & & \\
\hline i] & -6.08 & 0.20 & 0.102 & 0.09 & -0.09 & -0.25 & -0.11 & $0.5 .5 *$ & (0).7.7\%* & & & & & & & & & \\
\hline 11 & 0.32 & 0,07 & 0.18 & 0.15 & -0.15 & -0.13 & 0.28 & -0.27 & 0.13 & $0.45^{*}$ & & & & & & & & \\
\hline 12 & 0.37 & 0.01 & 0.18 & 0.10 & -0.10 & -0.09 & 0.34 & $-0) .15$ & -0.09 & 0.18 & $0.96^{* *}$ & & & & & & & \\
\hline 13 & 0.30 & -0.21 & 0.11 & 0.06 & -0.06 & 0.15 & 0.26 & 0.21 & -0.08 & -0.03 & 0.11 & 0.11 & & & & & & \\
\hline 14 & 0.17 & 0.37 & -0.12 & $0.2 !$ & -0.21 & 0.22 & -0.16 & 0.27 & -0.19 & -0.23 & 0.08 & 0.15 & -0.12 & & & & & \\
\hline 15 & $0.50 *$ & 0.06 & 0.00 & 0.11 & -0.11 & -0.34 & $0.68^{* *}$ & 0.14 & 0.05 & 0.26 & 0.26 & 0.22 & 0.16 & -0.01 & & & & \\
\hline 16 & 0.37 & 0.23 & 0.00 & -0.30 & 0.30 & -0.23 & 0.50 & 0.02 & -0.24 & -0.23 & -0.23 & -0.17 & 0.0 .3 & 0.17 & $0.43^{*}$ & & & \\
\hline 17 & -0.01 & 0.16 & $-(0.05$ & -0.41 & 0.41 & -0.08 & 0.04 & -0.14 & -0.29 & $-0.43^{*}$ & -0.42 & -0.32 & -0.12 & 0.17 & -0.28 & $0.73^{* *}$ & & \\
\hline 18 & 0.07 & 0.21 & -0.30 & 0.31 & -0.31 & -0.19 & -0.03 & 0.04 & 0.35 & 0.39 & 0.01 & -0.11 & 0.11 & 0.17 & 0.36 & 0.10 & -0.17 & \\
\hline 19 & 0.37 & 0.33 & -0.12 & -0.04 & 0.04 & -0.28 & $0.45^{*}$ & 0.07 & 0.03 & 0.08 & -0.09 & -0.12 & 0.09 & 0.24 & $0.66^{* *}$ & $0.83^{* *}$ & 0.38 & $0.60 * *$ \\
\hline
\end{tabular}

* Significant at $\mathrm{p}<0.05 ;{ }^{* * * *}$ Significant at $\mathrm{p}<0.01$.

Abbreviation: 1. Seed length, 2. Seed breadth, 3. Seed weight, 4. Healthy seed, 5. damage seed, 6. Germination, 7. Germination value, 8. Survival, 9. Plantable seedling, 10. Shoot length, 11. Root length, 12. Root/shoot ratio, 13. Collar diameter, 14. No of leaves, 15. Shoot dry weight, 16. Root dry weight, 17. Root/shoot dry weight, 18.leaves dry weight, 19.Total dry weight.

Table 5b. - Correlation coefficient between different seed and seedling and three geographical variables.

\begin{tabular}{|c|c|c|c|c|c|c|c|c|c|c|c|c|c|c|c|c|c|c|c|}
\hline $\begin{array}{l}\text { Geograplical } \\
\text { Alujbulus }\end{array}$ & I & 2 & 3 & 4 & 5 & 6 & 7 & 8 & $y$ & $\therefore$ & $\because 1$ & 12 & 13 & $1+$ & 1.5 & $: 6$ & 17 & 18 & 19 \\
\hline Altilude & (0. $49^{*}$ & a.17 & $0.68^{* *}$ & 0.39 & -0.39 & $0.45^{*}$ & 0.27 & 0.22 & $0.1 \mathrm{~A}$ & 0.08 & 0.31 & 0.31 & $0.13^{*}$ & $0.01 \mathrm{~A}$ & 0.088 & -0.29 & -10.42 & -0.12 & -0.23 \\
\hline 1.aticude & 0.39 & $-(0,11$ & 9. 12 & $-i, 10$ & 0,111 & -0.07 & 0.17 & 0.29 & $-\left(0.17^{*}\right.$ & $-[k, 32$ & -0.29 & $-(0,23$ & 0,71 & $-1,02$ & $0,0,8$ & 0.1 .1$. & a.6: & 0,03 & (1). 10 \\
\hline 1. (m) pitude & 0.3 .3 & 0.01 & .0 .618 & 0.34 & $-(1.3)$ & | & 0.196 & -0.06 & 0.32 & $0.4 .3 *$ & 0.24 & 0.10 & (2) 1.5 & 0.24 & 0.30 & 0.04 & -1).15 & 0.19 & (3).16 \\
\hline Rnitilall & (1).fig** & 9.11 & 0.11 & $0.97 * *$ & $-(1, \ldots) * * *$ & 0.018 & $0 .+1$ & 0.30 & -0.06 & 0.06 & 0.24 & 0.2 .3 & 0.04 & 0.19 & 0.1.** & -0.12 & $-0.48^{*}$ & 0.14 & \\
\hline $\mathrm{pH}$ & 0.13 & 0.17 & $0.57 * *$ & 0.35 & -0.36 & $0,67^{* *}$ & 0.18 & 0.15 & 0,05 & 0,02 & $0 . \therefore \mathrm{h}$ & i). 15 & 0.20 & -12.19 & $-(10,015$ & -0.26 & $-(10.27$ & $-(1)=0$ & -0.28 \\
\hline
\end{tabular}

* Significant at $\mathrm{p}<0.05 ;{ }^{* *}$ Significant at $\mathrm{p}<0.01$.

Abbreviation: 1. Seed length, 2. Seed breadth, 3. Seed weight, 4. Healthy seed, 5. Damage seed, 6. Germination, 7. Germination value, 8. Survival, 9. Plantable seedling, 10. Shoot length, 11. Root length, 12. Root/shoot ratio, 13. Collar diameter, 14. No of leaves, 15. Shoot dry weight, 16. Root dry weight, 17. Root/shoot dry weight, 18. Leaves dry weight, 19.Total dry weight.

Table 6. - Estimation of genetic parameters for seed, seedling and germination in laboratory condition.

\begin{tabular}{|c|c|c|c|c|c|c|c|c|c|c|c|c|c|c|c|}
\hline Parameters & 1 & 2 & 3 & 4 & 5 & 6 & 7 & 8 & 9 & 10 & 11 & 12 & 13 & 14 & 15 \\
\hline $\begin{array}{l}\text { Horitability } \\
\text { (Broad } \\
\text { sense) }\end{array}$ & 61.90 & 76.72 & 92.26 & 77.00 & 91.67 & 32.34 & 43.32 & 63.85 & 83.33 & 10.00 & 80.10 & 84.21 & 73.47 & 27.78 & 82.42 \\
\hline Genetic gain & 14.29 & 10.86 & 50.14 & 1.77 & 18.10 & 8.00 & 9.89 & 59.85 & 15.85 & 1.58 & 6.94 & 71.62 & 46.21 & 6.91 & 41.80 \\
\hline
\end{tabular}

Abbreviation: 1. Seed length, 2. Seed breadth, 3. Seed weight, 4. Germination, 5. Germination value, 6. Survival, 7. Shoot length; 8. Root length, 9. Root/shoot ratio, 10. Collar diameter, 11. No of leaves, 12. Shoot dry weight, 13. Root dry weight, 14. Root/shoot dry weight, 15 . leaves dry weight. 
Table 7. - Analysis of variance for various seed and seedling characteristics among and within the provenance of $Q$. leucotrichophora.

\begin{tabular}{|c|c|c|c|c|c|c|c|c|c|c|c|c|c|}
\hline \multicolumn{14}{|c|}{ Mcan Sum of Squares of sced and secdling characters } \\
\hline $\begin{array}{l}\text { Source of } \\
\text { variation }\end{array}$ & DI: & SI. & $\mathrm{SB}$ & SW & $0 \%$ & $\mathrm{GV}$ & SI & $\mathrm{R} L$. & $\mathrm{CI}$ & NOL & $\mathrm{S}[\mathrm{WW}$ & RISW & $113 \mathrm{~W}$ \\
\hline Population & 17 & $0.21^{* *}$ & $3.09^{* *}$ & $2462.0^{* *}$ & $93.85^{* *}$ & $0.33 * *$ & $28.21^{* *}$ & $225.56^{* *}$ & $0.24 * *$ & $2.55^{* *}$ & $0.07^{* *}$ & $0.53^{* *}$ & $1.76^{* * *}$ \\
\hline lumiliss & 4 & 0.01 & 0.01 & 26 & $\$ 4.69$ & 0,001 & 0.54 & 0.36 & 0.01 & 0.11 & 0.001 & 0.004 & 0.002 \\
\hline
\end{tabular}

** Significant at $(\mathrm{p}<0.01)$.

Abbreviations: $\mathrm{DF}=$ degree of freedom, $\mathrm{SL}=$ Seed length, $\mathrm{SB}=$ Seed breadth, $\mathrm{SW}=$ Seed weight, G\%=Germination percent, $\mathrm{GV}=$ Germination value, $\mathrm{SL}=$ Shoot length, $\mathrm{RL}=$ Root length, $\mathrm{CD}=$ Collar diameter, $\mathrm{NOL}=$ Number of leaves, SDW $=$ Shoot dry weight, RDW = Root dry weight, TDW = total dry weight.

was the highest $(71.62 \%)$ for shoot dry weight, followed by root length $(59.85 \%)$ and minimum $(0.95 \%)$ for shoot length (Table 6).

\section{Analysis of variance}

Analysis of variance between the provenance for different seed and seedling characteristics exhibited highly significant $(\mathrm{p}<0.01)$ variations. However, all these traits were not significantly different among the provenances (Table 7).

\section{Discussion}

The provenance of $Q$. luecotrichophora exhibited considerable amount of variation in morphological traits of seeds. Significant $(p<0.05)$ variations were obtained in seed length, breadth and weight among the provenances. The magnitude of variations was the highest for seed weight and the lowest for seed breadth (Table 2). These variations in seed morphological characters of $Q$. leucotrichophora may probably be due to resource availability, which varies over seasons and therefore, may influence the seed size (MURALI and SUKUMAR, 1994). The coefficients of variation were low with regard to seed size (5.85-9.17\%) while moderate for seed weight $(28.85 \%)$ and highest (63.48) for damaged seeds (Table 2). Similarly, difference in seed weight between provenances could have been a result of differences in the environmental conditions, e.g., nutrients, light or water to which the mother plants were subjected during the growing season (WOLFE, 1995) or due to different positions on mother plants, resulting in differences in seed filling (GUTTERMAN, 1992). Various researchers have reported that seed origin influence greatly seed and seedling characteristics of many species e.g. Grewia optiva (UNIYAL et al., 2002), Albizia chinensis (DHANAI et al., 2003), Celtis australis (SINGH et al., 2006), Cordia africana (LOHA et al., 2006), Dalbergia melanoxylon (AMri et al., 2008), Pinus roxburghii (GHILDIYAL et al., 2009), Quercus glauca (SiNGH et al., 2010). The seeds were collected from different locations, from trees approximately of the same age, therefore, differences observed in seed characters may be result of adaptation to diverse environmental conditions prevailing in their distribution range (MATHUR et al., 1984).

It has been observed that seed germination of $Q$. leucotrichophora is temperature dependent and constant temperature of $25^{\circ} \mathrm{C}$ induces the highest germination, irrespective of provenances. On average, 13 provenances out of 18 showed $<90 \%$ germination at $25^{\circ} \mathrm{C}$. Altitude affects the germination of this species. Significant $(p<0.05)$ variations have been recorded for germination response of provenances (Table 3). Germination of the seeds of $Q$. leucotrichophora is hypogeal with the nut splitting towards the apex and the radicle emerging and growing downward to form a young taproot. Similar finding was recorded in $C$. australis by SINGH et al. (2004). Several workers also reported that seed germination is temperature dependent and constant temperature $25^{\circ} \mathrm{C}$ accelerate germination in Albizia chinensis (DHANAI et al., 2003), A. lebbek (TODARIA et al., 2002) and Dalbergia sissoo (SINGH and BHATT, 2008a).

Significant $(p<0.05)$ variations have been recorded for growth of seedlings among provenances. The average shoot length was $34.96 \mathrm{~cm}$ for one year old seedlings, irrespective of provenance. Similarly, average root length was $28.12 \mathrm{~cm}$, irrespective of provenance after one year of growth. Significant variations were also obtained for collar diameter of seedlings of different provenances. Further, significant $(p<0.05)$ variations were also recorded in biomass allocation of seedlings among provenances. On average, roots partitioned the highest biomass followed by leaves and shoot.

The biomass of the seedlings, root length and root/shoot ratio exhibited moderate coefficient of variation while, shoot length, collar diameter and number of leaves exhibited lower values of coefficient of variation (Table 4). In an earlier study, NEGI and NAITHANI (1995) suggested that growth of $Q$. leucotrichophora seedling in forest stand is slow and during first year, the seedling may attain a height of only $5-12.5 \mathrm{~cm}$ with $2-5$ leaves per plant. During the course of present investigation, the seedling growth was comparatively higher and it may be attributed to the fact that, seeds were harvested from promising ideotypes which resulted into high seedling vigour. Several studies have been carried out to examine the variation in seedling traits among the seed sources in different species in Garhwal Himalaya viz. Terminalia tomentosa, T. bellerica, T. chebula (CHAUHAN et al., 2007), Grewia optiva (UNIYAL et al., 2002), Albizia chenensis (DHANAI et al., 2003), Celtis australis (SINGH and BHATT, 2008b) and the present findings are in conformity with the findings of these earlier workers.

Various workers have proved that seed size affect the seedling vigour (VERE, 1997; SINGH et al., 2006), however, growth of the seedlings in $Q$. leucotrichophora was not affected by seed size (Table $5 a$ ), but seed weight was significantly positively correlated with altitude indicat- 
ing that higher elevational seed source produce heavier seed in this species. Seedling growth traits, however, did not exhibit any positive or negative trend with altitude except collar diameter. Latitude of seed source was inversely correlated with plantable seedling indicating that these traits showed southern trend. The longitude exhibited positive correlation with shoot length indicating this trait increased towards eastern extremes. Similarly, soil $\mathrm{pH}$ was also significantly positive correlated with seed weight and germination indicating that higher soil $\mathrm{pH}$ was more suitable for production of vigour seeds.

The variance registered in $Q$. luecotrichophora for seedling growth and biomass parameters showed considerable variation. For the utilization of observed variation in species, it is a prerequisite to know the extent of variation and also that whether it is due to the genetic or the environmental factors. Hence information on variation among the desirable parameters and their correlation is vital for any breeding program (JOHNSON et al., 1955). Seed and seedling characteristics of $Q$. leucotrichophora exhibited a wide range of variation among provenances. On average, seed weight exhibited the highest heritability and genetic gain. This signified that seed weight is under strong genetic control (Table 3).

Higher values for broad sense heritability and genetic gain were estimated for seed weight, root length, shoot dry weight, root dry weight, leaves dry weight and moderately high for seed length, seed breadth, germination value, root/shoot ratio, indicating better chance of improving these characteristics at early stages through provenance and parent tree selection in $Q$. leucotrichophora. This supports earlier findings of SRIVASTAVA et al. (1993), GwAZE (1997), DHANAI et al. (2003) and SingH et al. (2008a). MANTOVEN (2002) also reported that variation on the provenance level in Prosopis flexuosa was related to the elevation, geographic location and some abiotic conditions of the seed source. DHILLON and KHAJURIA (1994) also recorded more than $75.0 \%$ heritability for various seed traits of Acacia nilotica. Moderate high heritability estimation with moderate genetic advance has earlier been reported for plant height of Terminalia arjuna (SRIVASTVA et al., 1993), plant height and stem diameter of Grewia optiva (SHARMA and SHARMA, 1995), Eucalyptus grandis (SuBRAMANIAN et al., 1995) and Dalbergia sissoo (GERA et al., 2000).

In general, heritability estimated along with genetic gain was reported more useful than the heritability alone in predicting the resultant effect for selecting the best genotypes for a given trait (VOLKER et al., 1990; SingH and CHAUdHARY, 1993). The observations incorporated in this investigation bring out fact that, seeds of $Q$. leucotrichophora collected from different provenances show large variation in seed morphology, seed weight, germination and seedling parameters. However, the variations were random between provenances. Therefore, all these characters should be considered as important traits for the selection because seedlings of seed lots selected on the basis of one character alone may some time not give the desired level of superiority. Seed source selection should be made on the basis of multi traits consideration as reported by GINEWAL et al. (1996) and VAKSHASYA et al. (1992).

Out of nineteen characters of seed and seedlings, ten characters were significantly correlated with geographical variables (altitude, latitude and longitude), and soil $\mathrm{pH}$ of the seed populations. The existing positive significant correlation between seed weight and altitude of seed source indicates a trend of increasing heavy seeds towards higher elevational sources which could be important criterion for seed source selection. Further more, higher germination was recorded in heavier seeds. Most of the provenances proved better germination at $25^{\circ} \mathrm{C}$. The middle to higher altitudinal population exhibited stable performance in seedling growth and biomass production indicating that species is most suitable for planting in middle to higher elevational ranges, where it is adapted genetically and physiologically. The characters, which showed greater influence, can be directly screened/selected for the improvement of this potential tree species, i.e., shoot dry weight, root length and seed weight. Root and leaf dry weight which indicate high heritability with moderately high genetic gain could be selected and considered for possibility of rapid genetic improvement of this potential tree at nursery stage to obtain quality seedlings for mass afforestation in Central Himalaya, India.

\section{References}

Amri, E., H. V. M. LyaruU, A. S. Nyomoral and Z. KANYEKA (2008): Effect of timing of seed collection and provenance on seed viability and germination of Dalbergia melanoxylon. Botany Research Journal 1: 82-88.

Andersen, U. S., J. P. P. Cordova, U. B. Nielsen and J. KOLLMANN (2008): Provenance variation in germination and seedling growth of Abies guatemalensis Rehder. Forest Ecology and Management 255: 1831-1840.

BARTZ, A. E. (1988): One-way Analysis of Variance. In: Basic Statistical Concepts, $3^{\text {rd }}$ edition, MacMilan Publishing Company, New York, pp. 278-303.

Chauhan, S., A. K. Negi and N. P. Todaria (1996): Effect of provenance variation and temperature on seed germination of Alnus nepalensis. Plant Physiology \& Biochemistry 23: 94-95.

Chauhan, S., Bhupendra Singh, B. P. Bhatt and N. P. TODARIA (2007): Effects of the altitude of seed origin and storage on germination of three Terminalia species, Garhwal Himalaya, India. Forests Trees and Livelihoods 17: 339-344.

CZABATOR, F. J. (1962): Germination value: An index combining speed and completeness of pine seed germination. Forest Science 8: 386-396.

Dhanai, C. S., A. K. UniYal and N. P. TODARIA (2003): Source Variation in Albizia chinensis (OSBECK) MER. Seed and seedling characteristics. Silvae Genetica 52(5-6): 259-266.

Dhillon, G. P. S. and H. N. KhaJuRIA (1994): Genotypic variation in germination and potential in Acacia nitoli$c a$ (C.) seeds. Indian Journal of Forestry 17(4): 345-347.

GAUR, R. D. (1999): Flora of the District Garhwal: Northwest Himalaya with Ethnobotanical Notes. Transmedia Publication Center, Srinagar Garhwal, Uttaranchal, India, pp. 811. 
Gera, M., N. Gera and B. N. Gupta (2000): Preliminary observations on genetic variability and character association in Dalbergia sissoo Roxb. Indian Forester 134: 608-615.

Ghildiyal, S. K., C. M. Sharma and S. Gairola (2009): Additive genetic variation in seedling growth and biomass of fourteen Pinus roxburghii provenances from Garhwal Himalaya. Indian Journal of Science and Technology 2: 37-45.

Ginwal, H. S., M. Gera and R. L. Srivastava (1996): Seed source variability in some seed and seeding characteristics of twenty provenances of Acacia nilotica WILLD. Ex. DEL. Range Management and Agroforestry 17(1): 49-59.

GutTERMAN, Y. (1992): Maternal effects on seed during development. pp. 27-59. Seeds. The Ecology of Regeneration in Plant Communities. In: FENNER, M. (ed.) Wallingford CAB International.

GwAZE, D. P. (1997): Genetic parameter estimate for height and stem straightness in Pinus taeda L. and implications for breeding. Ph.D. Thesis, University of Edinburgh, pp. 180.

Holm, S. O. (1994): Reproductive patterns of Betula pendula and B. pubescens Coll. along a regional altitudinal gradient in Northern Sweden. Ecography 17: 60-72.

IsIK, K. (1986): Altitudinal variation in Pinus brutia Ten: seed and seedling characteristics. Silvae Genetica 35: 59-67.

ISTA (1999): International Rules for Seed Testing. Seed Science and Technology, 27, Supplement, Zurich, Switzerland.

Johnson, H.W., H. F. Robinson and R. F. Comsstock (1955): Estimates of genetic and environmental variability in soyabean. Agronomy Journal 47: 314-318.

Loha, A., M. Tigabu, D. Teketay, K. Lundkvist and A. FRIES (2006): Provenance variation in seedmorphometric traits, germination, andseed ling growth of Cordia africana Lam. New Forests 32: 71-86.

Mantovan, N. G. (2002): Early growth differentiation among Prosopis flexuosa D.C. provenances from the Monte phytogeographic province, Argentina. New Forest 23(1): 19-30.

Mathur, R. S., K. K. Sharma and M. M. S. Rawat (1984): Germination behaviour of various provenances of Acacia nilotica subspecies indica. Indian Forester 110(5): 435-449.

Murali, K. S. and R. Sukumar (1994): Reproductive phonology of a tropical dry deciduous forest in Southern India. Journal of Ecology 82: 759-767.

Mwase, W. F., A. BJornstad, Y. M. Ntupanyama, M. B. KWAPATA and J. M. BoKosi (2006): Phenotypic variation in fruit, seed and seed ling traits of nine Uapaca kirkiana provenances found in Malawi. Southern Forests: A Journal of Forest Science 208(1): 15-21.

NeGi, S. S. and H. B. NAithani (1995): Oaks of India, Napal and Bhutan. International Book Distributors, 9/3 Rajpur Road Dehra Dun India pp. 166.

SACHAN, M. S. (2007): Structure and Functioning of traditional agroforestry systems along an altitudinal gradient in Garhwal Himalaya“. Ph. D. thesis submitted to HNB Garhwal University, Srinagar (Garhwal). Uttarakhand.

SAikia, S. P., B. S. BhaU, A. RabHA, S. P. DUtTA, R. K. Choudhari, M. Chetia, B. P. Mishra and P. B. KanjILAL (2009): Study of accession source variation in morphophysiological parameters and growth performance of Jatropha curcas Linn. Current Science 96: 1631-1636.
SchmidT, L. (2000): Guide to Handling of Tropical and Subtropical Forest Seed. Danida Forest Seed Center, Denmark, pp. 511.

SHARMA, R. K. and S. S. SHARMA (1995): Heritability and association analysis in Grewia optiva. Indian Forester 121(4): 318-320.

Singh, B., K. P. SakLani and B. P. Bhatt (2010): Provenance variation in seed and seedling attributes of Quercus glauca Thunb in Garhwal Himalaya, India. Dendrobiology 63: 59-63.

Singh, Bhupendra and B. P. BhATt (2008a): Provenance variation in pod, seed and seedling traits of Dalbergia sissoo Roxb., Central Himalaya, India. Tropical Agricultural Research \& Extension 11: 39-44.

Singh, BHUPENDRA and B. P. BHATT (2008b): Variability in seed and seedling traits of Celtis australis in Central Himalaya, India. Plant Genetic Resources News Letters 156: $56-61$.

Singh, Bhupendra, B. P. Bhatt and P. Prasad (2004): Effect of seed source and temperature on seed germination of Celtis australis L.: a promising agroforestry treecrop of Central Himalaya, India, Forests, Trees and Livelihoods 14: 53-60.

Singh, Bhupendra, B. P. Bhatt and P. Prasad (2006): Variation in seed and seedling traits of Celtis australis, a multipurpose tree, in Central Himalaya, India. Agroforestry Systems 67: 115-122.

Singh, N. B. and V. K. ChaudhaRY (1993): Variability, heritability and genetic gain in cone and nut characters of Chilgoza Pine (Pinus gerardiana. Wall). Silvae Genetica 42: 2-3.

Srivastava, D. P., P. K. SRivastava, A. Goal and K. ThangaVelu (1993): Genetic variability in Terminalia arjuna Bedd. Indian Journal Forestry 16(3): 223-225.

Subramanian, K. N., A. K. Mandal and A. I. Nicodemus (1995): Genetic variability and character association in Eucalyptus grandis. Annals of Forestry 3(2): 134-137.

TOdARIA, N. P., BHUPENDRA Singh and RAM KumAR (2002): Seed source variation and germination studies in Albizia lebbek from Garhwal Himalaya. Journal of Tree Science 21: 27-34.

UniYal, A. K., B. P. Bhatt and N. P. TOdaria (2002): Provenance variation in seed characteristics of Grewia oppositifolia Roxb. - A Promising agroforestry tree-crop of Central Himalaya. India. Indian Journal of Forestry 25(2): 209-214.

VAKshasya, R. K., O. P. Rojora and M. S. RaWAT (1992): Seed and seedling traits of Dalbergia sissoo Roxb: Seed source variation studies among ten provenances in India. Forest Ecology and Management 48: 265-275.

VERA, M. L. (1997): Effects of altitude and seed size on germination and seedling survival of healthland plants in north Spain. Plant Ecology 133: 101-106.

Volkar, P. W., C. A. Dean, W. B. Tibbits and I. C. RauenWOOD (1990): Genetic parameters and gain expected from selection in Eucalyptus globulus in Tasmania. Silvae Genetica 39(1): 18-21.

WoLfE, L. M. (1995): The genetics and ecology of seed size variation in a biennial plant Hydrophyllum apendiculatum. Oecologia 101: 343-352.

Zheng, Y. L., W. B. Sun, Y. Zhou and D. Coombs (2009): Variation in seed and seedling traits among natural populations of Trigonobalanus doichangensis (A. Camus) Forman (Fagaceae), a rare and endangered plant in southwest China. New Forests 37: 285-294. 